\title{
Immunohistochemical Expression of Fibroblast Activation Protein (FAP) in Endometrial Carcinoma Subtype at The Department of Anatomical Pathology Faculty of Medicine Universitas Sumatera Utara / RSUP H. Adam Malik Medan
}

\author{
${ }^{1}$ Fatma Zulita Fiandani, ${ }^{2}$ Delyuzar, ${ }^{3}$ Soekimin, ${ }^{4}$ Joko S Lukito, ${ }^{5}$ Jessy Chrestella
}

Email address: mrs.muhar@yahoo.com

${ }^{1}$ Department of Anatomical Patholog, Faculty of Medicine, Universitas Sumatera Utara Medan, Indonesia

Abstract

Background: Endometrial cancer (EC) is the most commonly diagnosed gynecologic malignancy globally; The etiology of EC is still unknown, but most of the risk factors are related to changes in hormonal patterns that occur in women. In the female reproductive tract, fibroblasts promote epithelial development and differentiation which responsible for extracellular matrix remodeling, survival and death. Fibroblast activation protein (FAP) is a cell surface antigen that expressed on reactive stromal fibroblasts of cancer epithelial cells and appears as an important factor in cancer-associated fibroblasts (CAF), which relevant in tumor development. FAP has effects on cell proliferation, migration, and invasion, which promote tumor growth. To date, only one study of FAP has been found on the endometrium. In addition, there have been no studies on the expression of FAP against endometrial carcinoma subtypes.

Objective: This study was conducted to analyze the immunohistochemical expression of FAP in endometrial carcinoma sub subtypes.

Methods: The study used 38 samples of paraffin blocks from endometrial carcinoma cases that had been diagnosed at the Laboratory of Anatomical Pathology, RSUP H. Adam Malik and the Department of Anatomical Pathology, Faculty of Medicine, Universitas Sumatera Utara. FAP expression was assessed using immunohistochemical staining of tumor cell cytoplasm and stroma. Expressions were assessed using the Quick Score method and were declared positive if the total score $\geq 3$.

Results: Immunohistochemical expression of FAP in endometrial carcinoma was the most positive in the endometrioid carcinoma subtype, namely 27 cases $(77.14 \%)$.

Keywords: endometrial carcinoma, cancer-associated fibroblasts, fibroblast activation protein

\section{Introduction}

Endometrial carcinoma (EC) is a malignancy in the field of gynecology with an increasing incidence every year. According to World Health Organization (WHO) data in 2012, EC was the sixth most common cancer found in Indonesian women, while in the world it was the fifth highest. And will further increase to $50 \%$ by 2040 worldwide. ${ }^{1}$ In the United States, EC ranks fourth, and is the most common genital malignancy. Whereas in countries such as India and Southeast Asia the incidence is lower. ${ }^{2}$ Every year worldwide about 142,000 women are diagnosed with endometrial carcinoma, and 42,000 of them die from this disease. Incidence rates vary widely; The incidence of standard age is higher in North America and much of Europe than in the rest of the world. ${ }^{3}$ The exact etiology of endometrial carcinoma is still unknown, but most of the risk factors are related to changes in hormonal patterns that occur in women $[2,4]$.

Histologically, endometrial carcinoma is divided into endometrioid and non-endometrioid carcinoma. Where these two types of carcinoma differ in etiology and malignancy. Endometrioid carcinoma is the most common type, accounting for about $75-80 \%$ of all endometrial cancer cases, while clear cell carcinoma is only about $1-2 \%$ of all endometrial cancer cases $[2,8]$

Stromal cells communicate with each other with cancer cells and immune cells directly through cell-to-cell contact and indirectly through paracrine signaling, protease secretion, and extracellular matrix modulation. This complex communication network is essential for the microenvironment that supports tumorigenesis, angiogenesis, and metastasis. Fibroblasts are an important component of the tumor microenvironment, and can promote the growth and invasion of cancer cells through the synthesis, deposition, and remodeling of the extracellular matrix (ECM), which is involved in angiogenesis and deregulation of the antitumor immune 
response. Fibroblast Activation Protein (FAP) is emerging as an important factor in the pro-oncogenic function of these stromal cells, although further studies are needed to determine the mechanisms involved [6].

FAP was first discovered in 1986 by Wolfgang Rettig as a cell surface antigen expressed on reactive stromal fibroblasts of cancer epithelial cells. FAP is a type II serine membrane protease derived from the prolylcleaving peptidase family, including dipeptidyl petidases 4 (DPP4), DPP8, DPP9, and DPP2. FAP is the most homologous serine protease with DPP4, with 70\% identical amino acid sequence identity [7-9].

FAP is known to be overexpressed in breast, colorectal, pancreatic, lung, bladder, ovarian, and other cancers and usually highly expressed in the stroma and thus has become a universal marker of CAF. The overexpression of FAP is associated with poor prognosis and survival rates, as well as rapid progression of cancer. Although the presence of FAP in malignant tissue is unquestionable, the biological role of FAP and its impact on disease prognosis are inconsistent across the literature. [10].

Although studies of FAP have been carried out on several organs, to date, the authors have only found one study of FAP performed on the endometrium. In addition, there have been no studies on the expression of FAP against endometrial carcinoma subtypes. Therefore, we want to know and understand the immunohistochemical expression of FAP in endometrial carcinoma subtypes.

\section{Materials and Methods}

This study is a descriptive study that aims to assess the expression of FAP in endometrial carcinoma subtypes with a cross-sectional approach where each sample in this study was observed once at a time. The study used 38 samples of paraffin blocks from endometrial carcinoma cases that had been diagnosed at the Laboratory of Anatomical Pathology, RSUP H. Adam Malik and the Department of Anatomical Pathology, Faculty of Medicine, Universitas Sumatera Utara. The research has obtained permission from the Ethics Committee for Health Research Implementation, Faculty of Medicine, Universitas Sumatera Utara, Medan with no: $233 / \mathrm{KEP} / \mathrm{USU} / 2021$. This study displays the frequency distribution of patients with endometrial carcinoma by age and based on its hystopathological subtypes at the Department of Anatomic Pathology, Faculty of Medicine, Universitas Sumatera Utara / H. Adam Malik Hospital, Medan.

We used a sample that had the inclusion and exclusion criteria. Where the inclusion criteria were slides and paraffin blocks derived from post-hysterectomy tissue diagnosed histopathologically EC by hematoxylin-eosin (HE) staining. Meanwhile, the exclusion criteria are missing or damaged paraffin slides and blocks that cannot be re-evaluated and after the review is not an endometrial carcinoma.

The proportion score of the area of the stained cells was divided into: $0 \leq 10 \%, 1=11-25 \%, 2=26-50 \%$, $3=>51 \%$. The staining intensity score was divided into: $0=$ negative (unstained), $1=$ weak positive, $2=$ moderate positive, $3=$ strong positive. The final result is by adding up the area proportion score and the intensity score and is interpreted as a positive if the final score is $\geq 3$ [23].

\section{Results}

In this study, 38 samples of EC were found that met the inclusion and exclusion criteria at the $\mathrm{H}$ Adam Malik General Hospital, Medan. Frequency distribution by age, histopathological subtype, and FAP expression in endometrial cancer subtypes.

Data on the frequency distribution of patients with endometrial carcinoma based on age in this study had a mean age of 55.5 years with a standard deviation of 11.4 years, with the youngest age are 31 years old and the oldest are 84 years old. The age distribution of endometrial carcinoma patients was mostly found in the age group > 55 years, namely 20 cases (52.6\%), followed by the 46-55 year age group with 12 cases (31.6\%), and the least in the age group are 45 years old as many as 5 cases $(15.8 \%)$. Meanwhile, based on the histopathological subtype, endometrioid carcinoma was the most common subtype in this study, as many as 35 cases $(92.1 \%)$. The second most common subtype was clear cell carcinoma with 2 cases $(5.2 \%)$. Serous carcinoma subtype as many as 1 case (2.6\%). Meanwhile, the other subtypes, namely mucinous carcinoma, undifferentiated carcinoma and dedifferentiated carcinoma, were not found in this study. The immunohistochemical expression of FAP was found in 28 cases $(73,7 \%)$, while the negative expression was found in 10 cases $(26,3 \%)$. (Table 1$)$. 
Table 1. Frequency distribution of endometrial carcinoma patients based on age, histopathological subtype and FAP expression in endometrial cancer.

\begin{tabular}{lcc}
\hline \multicolumn{1}{c}{ Sample characteristics } & Total $(\mathbf{n})$ & Pecentage (\%) \\
\hline Age (years) & 6 & 15,8 \\
$\leq 45$ years & 12 & 31,6 \\
$46-55$ years & 20 & 52,6 \\
$>55$ years & & \\
Histopathological subtype & 35 & $92,1 \%$ \\
Endometrioid carcinoma & - & - \\
Mucinous carcinoma & 1 & $2,6 \%$ \\
Serous carcinoma & 2 & $5,3 \%$ \\
Clear cell carcinoma & - & - \\
Mixed cell carcinoma & - & - \\
Undifferentiated carcinoma & & $26,3 \%$ \\
Expression of FAP & 10 & $73,7 \%$ \\
Negative & 28 & $\mathbf{1 0 0}$ \\
Positive & $\mathbf{3 8}$ & \\
& &
\end{tabular}

In this study, the most positive FAP expression was shown in the endometrioid carcinoma subtype, namely 27 cases $(77.14 \%)$, followed by the clear cell carcinoma subtype, which was 1 case $(50 \%)$. While in serous carcinoma subtype, nothing was shown. (Table 2).

Table 2. Distribution of FAP expression by histopathological subtype of endometrial carcinoma

\begin{tabular}{|c|c|c|c|c|c|}
\hline & & $\begin{array}{c}\text { Endometrioid } \\
\text { carcinoma }\end{array}$ & $\begin{array}{l}\text { Clear Cell } \\
\text { carcinoma }\end{array}$ & $\begin{array}{c}\text { Serous } \\
\text { carcinoma }\end{array}$ & Total \\
\hline FAP & Negative & 8 & 1 & 1 & 10 \\
\hline \multirow[t]{3}{*}{ expression } & & $(22.86 \%)$ & $(50 \%)$ & $(100 \%)$ & $(26,32 \%)$ \\
\hline & Positive & 27 & 1 & 0 & 28 \\
\hline & & $(77,14 \%)$ & $(50 \%)$ & $(0 \%)$ & $(73,68 \%)$ \\
\hline \multirow[t]{2}{*}{ TOTAL } & & 35 & 2 & 1 & 38 \\
\hline & & $(100 \%)$ & $(100 \%)$ & $(100 \%)$ & $(100 \%)$ \\
\hline
\end{tabular}



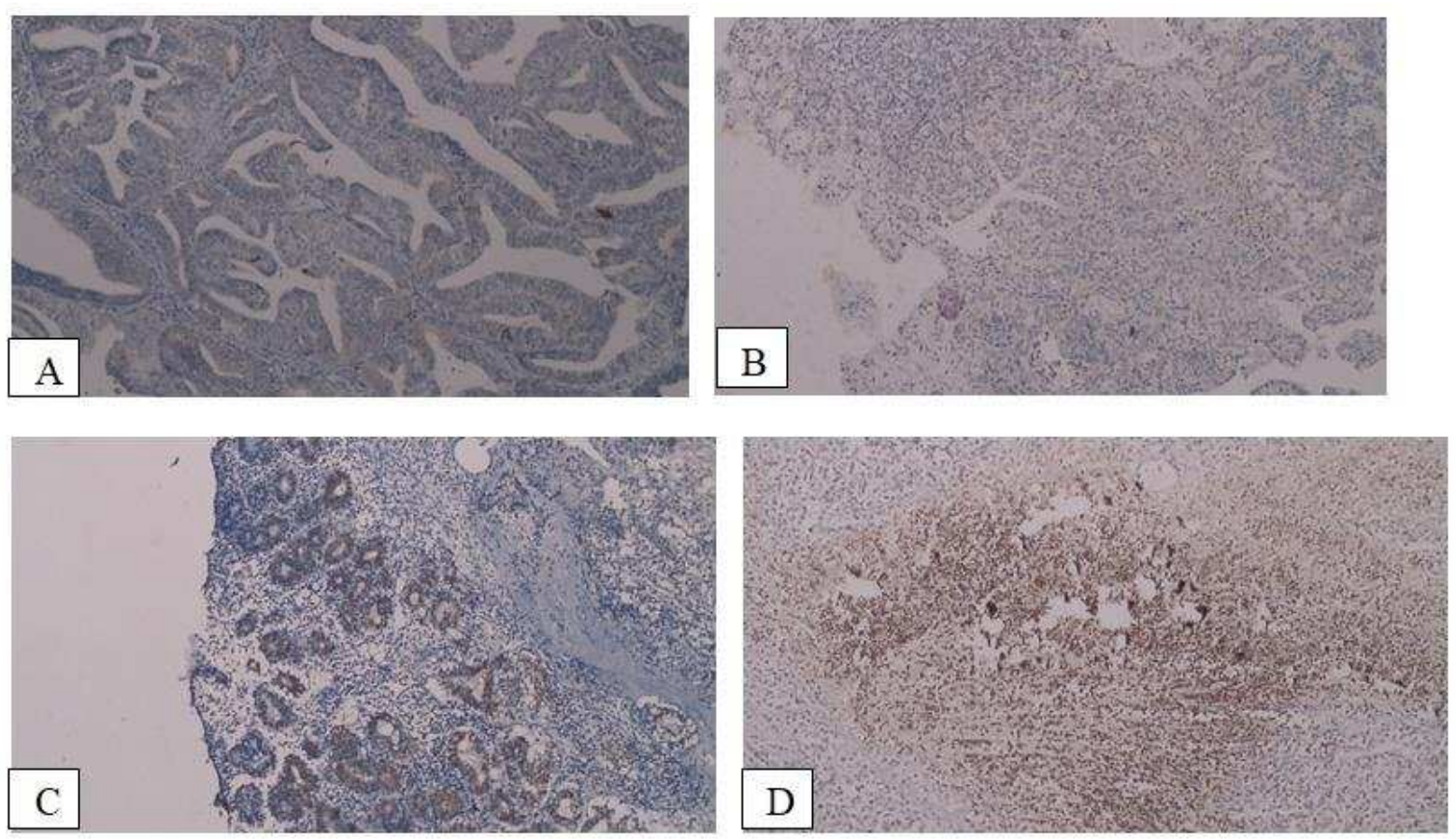

Figure 1. Microscopic examination. A-B. Weak FAP expression. C-D. Strong FAP expression.

\section{Discussion}

Endometrial carcinoma samples in this study were 38 samples recorded in the data storage software in the medical records of Anatomical Pathology Unit RSUP H. Adam Malik Medan which met the inclusion and exclusion criteria.

According to WHO, the risk of endometrial carcinoma increases in older women [11]. This is in accordance with the findings in this study where the most age group was $>55$ years, with a mean age of 55 years. This study is in line with the research conducted by Li et al. which divides endometrial cancer cases into 3 age groups, namely the 55-64 year age group which is the age group with the highest proportion, followed by the 65 years and <55 years age group [12]. The Women's Health Initiative (WHI) also published that the incidence of endometrial cancer increased over time in women aged 50-74 years (annual percentage change 2004-2009: 2.8\%; 2005-2009: 3.3\%; and 2006-2009 : 4.2\%) [13]. Likewise with the Contastine study in 2019 which stated that the global prevalence of endometrial cancer in $2010-2014$ was most commonly found in pre and post-menopausal women with the average age at diagnosis at the age of 64 years (>55 years) [14]. This may be due to prolonged exposure to estrogen, and the shift in the balance of progesterone and estrogen that occurs at menopause, with decreased production of progesterone, even small amounts of circulating estrogen may not be adequately balanced, leading to thickening of the endometrium and possibly subsequent endometrial cancer. Therefore, an increased risk of endometrial cancer also occurs in postmenopausal women who use hormone replacement therapy containing estrogen without a combination with progesterone $[11,15,16]$.

The youngest age of patients with endometrial carcinoma found in this study was at the age of 31 years. This may be due to the occurrence of chronic anovulation (in polycystic ovarian syndrome) due to the high endogenous estrogen levels that are common in obesity. In addition, mismatch repair abnormalities and Lynch syndrome are said to be associated with the pathogenesis of endometrial carcinoma at a young age $[16,17]$. Fadhlaoui et al. reported a case of endometrial carcinoma in a 27-year-old nulliparous woman with a history of obesity and infertility. This is consistent with the hypothesis that the stimulatory effect of estrogen on the endometrium, if not counteracted by progesterone, can induce endometrial carcinogenesis [18]. The study by Son J et al. in 2019 retrospectively studied endometrial carcinoma patients treated from January 2004 to August 2016 and found that out of a total of 551 patients, 103 of them were 40 years old (18.7\%). Where in this study, patients aged $\leq 40$ years were associated with an excess body mass index [19]. Because endometrial carcinoma is rare in young women, the diagnosis during the reproductive years should be made with caution, as grade 1 endometrial carcinoma may mimic a state of severe atypical hyperplasia. In younger patients, an underlying estrogen-related condition such as granulosa cell tumor, polycystic ovaries, or obesity may be considered [20].

Based on the 2014 WHO classification, endometrial carcinoma is divided into several subtypes according to histopathological features. However, of all these subtypes, in this study, researchers only found three subtypes, 
which were dominated by endometrioid carcinoma subtypes at $94.7 \%$. And there are two other histopathological subtypes, namely serous carcinoma and clear cell carcinoma. This study is in accordance with the literature which states that endometrioid carcinoma is the most common subtype of endometrial carcinoma which is about $89 \%$, followed by serous carcinoma subtype around $4 \%$ and clear-cell carcinoma subtype as much as $1 \%$ [21]. Subramaniam et al. also mentioned that endometrioid carcinoma represents the majority of sporadic cases, which is about $70-80 \%$ of endometrial carcinomas [22].

In recent years, research on the relationship between cancer and the tumor microenvironment related to growth, invasion and metastasis has increased. Research has shown that stromal fibroblasts, also known as CAFs, can support tumorigenesis and tumor progression through many mechanisms, including proliferation, angiogenesis, invasion, tumor survival and immune system suppression. Subsequent studies have shown that FAP, which is a major marker of CAF, plays an important role in the progression of many tumors. FAP is expressed in reactive CAF in the stroma and granulation tissue to support the wound healing process, but this FAP is not expressed in normal adult tissue, so this FAP can be used to estimate the prognosis of a tumor. In this study, FAP was expressed mainly in the stroma and partially in tumor cells. This is in accordance with research conducted by Jing Song, et al. in 2011 in endometrial carcinoma in which FAP was expressed mainly in the stroma $(89.9 \%)$ [23].

In this study also found that the highest histopathological subtype of FAP expression was in endometrioid carcinoma. There has never been a study that assessed the expression of FAP in histopathological subtypes of endometrial carcinoma. The highest proportion of FAP expression was endometrioid carcinoma because the largest sample in this study was that subtype. This study showed that FAP is also partially expressed in tumor cells. This result is in accordance with the research of Fang Liu et al. who stated that FAP in endometrial carcinoma can also be expressed in tumor cells other than in the stroma [23].

\section{Conclusion}

After conducting research on 38 samples of endometrial carcinoma, it can be concluded that the age frequency of patients with endometrial carcinoma is the most in the age group > 55 years $(52.6 \%)$. Based on the histopathological subtype, endometrioid carcinoma was the most common subtype in this study, with 35 cases (92.1\%). Immunohistochemical expression of Fibroblast Activation Protein (FAP) in endometrial carcinoma was the most positive in the endometrioid carcinoma subtype, namely 27 cases $(77.14 \%)$.

\section{Acknowledgements}

We would acknowledge to all staf and resident of Anatomical Pathology Department of Universitas Sumatera Utara and H. Adam Malik hospital, Medan, Indonesia for their help and cooperation.

\section{References}

1. Morice P, Leary A, Creutzberg C, Abu-Rustum N, Darai E. Endometrial cancer. Lancet [Internet]. 2016;115. Available from: http://dx.doi.org/10.1016/S0140-6736(15)00130-0

2. Patni R, editor. Current concepts in endometrial cancer. Springer US; 2017. 1-131 p

3. Felix AS, Yang HP, Bell DW, Sherman ME. Epidemiology of endometrial carcinoma: Etiologic importance of hormonal and metabolic influences. Vol. 943, Advances in Experimental Medicine and Biology. 2017. 3-46 p.

4. Campos S, Lee L, Del Carmen M, Mcmeekin D. Corpus: Epithelial Tumors. In: Chi DS, Berchuck A, Dizon DS, Yashar C, editors. Principles and Practice of Gynecologic Oncology. Seventh Ed. Wolters Kluwer; 2017. p. 984-1041

5. Cassey M, Summers G, Crotzer D. Endometrial Cancer. 2020; Available from: https://www.ncbi.nlm.nih.gov/books/NBK525981/

6. Liu R, Li H, Liu L, Yu J, Ren X. Fibroblast activation protein: A potential therapeutic target in cancer. Cancer Biol Ther. 2012;13(3):123-9

7. Li M, Cheng X, Rong R, Gao Y, Tang X, Chen Y. High expression of fibroblast activation protein (FAP) predicts poor outcome in high-grade serous ovarian cancer. BMC Cancer. 2020;20(1):4-11.

8. Fitzgerald AA, Weiner LM. The role of fibroblast activation protein in health and malignancy. Cancer Metastasis Rev. 2020;39(3):783-803.

9. Chung KM, Hsu SC, Chu YR, Lin MY, Jiaang WT, Chen RH, et al. Fibroblast activation protein (FAP) is essential for the migration of bone marrow mesenchymal stem cells through RhoA activation. PLoS One. 2014;9(2):1-11. 
10. Hamson EJ, Keane FM, Tholen S, Schilling O, Gorrell MD. Understanding fibroblast activation protein (FAP): Substrates, activities, expression and targeting for cancer therapy. Proteomics - Clin Appl. 2014;8(5-6):454-63.

11. Zaino R, Carinelli S., Ellenson L., Eng C, Katabuchi H, Konishi I, et al. Tumours of The Uterine Corpus. In: Kurman RJ, Carcangiu ML, Herrington S, Young RH, editors. WHO classification of tumours of female reproductive organs. 4th Editio. Lyon: International Agency for Researcher on Cancer (IARC); 2014. p. 124-54.

12. Li Z, Joehlin-Price AS, Rhoades J, Ayoola-Adeola M, Miller K, Parwani AV, et al. Programmed Death Ligand 1 expression among 700 consecutive endometrial cancers: strong association with mismatch repair protein deficiency. Int J Gynecol Cancer. 2017; 00: 1-10.

13. Rossouw JE, Anderson GL, Prentice RL, et al. Risks and benefits of estrogen plus progestin in healthy postmenopausal women: Principal results From the Women's Health Initiative randomized controlled trial. JAMA 2002;288:321-333.

14. Contastine $\mathrm{G}$ et al. Increased Incidence of Endometrial Cancer Following the Women's Health Initiative: An Assessment of Risk Factors. Journal Of Women's Health. 2019;28(2):237-242.

15. Mutter G, Guiu X, Lax S. Endometrial Adenocarcinoma. In: Robboy's Pathology of the Female Reproductive Tract. 2nd Editio. Churchill Livingstone: Elsevier; 2009. p. 393-426.

16. Pandia WN. Ekspresi imunohistokimia CD34 dan Vascular Endothelial Growth Factor (VEGF) pada kanker endometrium dan hiperplasia endometrium.2019. available from: http://repositori.usu.ac.id/handle/123456789/25487

17. Lekhi A, Manchanda R, Jain N, Chithra S, Kausar H. Endometrial carcinoma in young women: management options and its review. Int J Reprod Contracept Obstet Gynecol. 2016 Apr;5(4):944-947.

18. Fadhlaoui A, Hassouna JB, Khrouf M, Zhioua F, Chaker A. Case report: Endometrial Adenocarcinoma in 27 year old woman. Clinical Medicine Insights: Case Reports 2010:3 31-39.

19. Son J, et al. Endometrial cancer in young women: prognostic factors and treatment outcomes in women aged $\leq 40$ years. Int J Gynecol Cancer 2020;30:631-639. doi:10.1136/ijgc-2019-001105

20. Amant F, Mirza MR, Koskas M, Creutzberg CL. Cancer of the corpus uteri. Figo cancer report 2018. Int J Gynecol Obstet 2018; 143 (Supp12): 37-50.

21. Fadare O, Roma AA. Atlas of Uterine Pathology. Switzerland: Springer; 2019. 265 p.

22. Subramaniam KS, et al. Cancer-Associated Fibroblasts Promote proliferation of Endometrial Cancer Cells. PLoS One. Public Library of Science. 2013(8):pp.1-16.

23. Liu F, Qi L, Liu B, Liu J, Zhang H, Che DH, et al. Fibroblast activation protein overexpression and clinical implications in solid tumors: A meta-analysis. PLoS One. 2015;10(3):1-18. 